\title{
Cortisol stress responses and children's behavioral functioning at school
}

\author{
Sterre S.H. Simons | Antonius H.N. Cillessen | Carolina de Weerth
}

Behavioural Science Institute, Radboud University, Nijmegen, The Netherlands

Correspondence

Sterre S.H. Simons, Behavioural Science Institute, Radboud University, Postbus 9104 6500 HE Nijmegen, The Netherlands.

Email: s.simons@psych.ru.nl

Funding information

The Netherlands Organisation for Scientific Research (NWO), Grant number: 452-04-320; Behavioural Science Institute
The present study investigated whether cortisol stress responses of 6-year-olds were associated with their behavioral functioning at school. Additionally, the moderating role of stress in the family environment was examined. To this end, 149 healthy children ( $M_{\text {age }}=6.09$ years; 70 girls) participated in an age-appropriate innovative social evaluative stress test. Saliva cortisol samples were collected six times during the stress test to calculate two indices of the cortisol stress response: cortisol stress reactivity and total stress cortisol. Teachers assessed children's internalizing, externalizing, and prosocial behaviors. Stress in the family environment was operationalized as maternally reported parenting stress. Results indicated a significant increase in cortisol concentrations in response to the stressor. No significant associations were found between cortisol stress responses and behavioral functioning at school and there was no evidence for moderation by maternal parenting stress. Potential theoretical and methodological explanations for these results are discussed.

\section{KEYWORDS}

cortisol stress responses, family stress, behavior, teacher report, children

\section{1 | INTRODUCTION}

Normally developing children differ in how they behave at school. Behavioral functioning at school influences a child's peer relations and learning, and in turn the child's future. This study aimed to obtain more insight in the correlates of behavioral functioning at school by focusing on the role of children's cortisol stress responses. Furthermore, the moderating role of stress in the family environment in the form of maternal parenting stress was studied.

In stressful situations the hypothalamic-pituitary-adrenal (HPA) axis, with its primary hormonal end product cortisol, becomes activated. The HPA-axis prepares the individual to respond behaviorally and physiologically to a stressor. There are individual differences in this normative HPA-axis response (Kudielka, Hellhammer, \& Wüst, 2009). Moreover, both HPA-axis functioning and behavioral tendencies appear to be trait-like characteristics of a person (e.g., high vs. low reactive phenotype; Boyce \& Ellis, 2005).
Links between HPA-axis functioning and trait-like behavior in different contexts have been described. For example, in 4.5-year-olds higher cortisol reactivity in an emotion eliciting task, was associated with more mother reported dispositional effortful control (Spinrad et al., 2009). In 4- to 5-year-olds, a moderate increase in cortisol followed by down regulation was associated with more self-regulation, while higher baseline cortisol and blunted cortisol reactivity/decreasing cortisol was associated with more aggression (Blair, Granger, \& Razza, 2005). Higher cortisol reactivity was associated with less school engagement, less academic competence, and more externalizing behavior (boys only) in 5- to 6-year-olds (Obradović, Bush, Stamperdahl, Adler, \& Boyce, 2010). In 11-year-olds, lower cortisol reactivity was associated with more externalizing behaviors and delinquency whereas higher cortisol reactivity was associated with positive student-teacher relationships (Conradt et al., 2014). Others found no association between cortisol reactivity and impulsivity, internalizing, and externalizing behavior (e.g., Alink et al., 2008; Spinrad et al., 2009). In sum, this indicates that to date

This article was published online on 24 Sep 2016. An error was subsequently identified. This notice is included in the online and print versions to indicate that both have been corrected 4 Nov 2016.

The copyright line for this article was changed on 7 February 2017 after original online publication.

This is an open access article under the terms of the Creative Commons Attribution-NonCommercial-NoDerivs License, which permits use and distribution in any medium, provided the original work is properly cited, the use is non-commercial and no modifications or adaptations are made.

(c) 2016 The Authors. Developmental Psychobiology Published by Wiley Periodicals, Inc. 
findings regarding associations between child cortisol stress responses and trait-like behavioral functioning are varied and somewhat inconsistent.

These inconsistencies may be explained by moderators. One potential moderator may be stress in children's social environment. Quellet-Morin et al. (2011) showed that lower cortisol responses were associated with more behavioral and social problems, but only in bullied and/or maltreated children. Moreover, the interaction between family environment and cortisol reactivity predicted prosocial behavior. More family adversity was associated with less prosocial behavior but only in high cortisol reactive children (Obradović et al., 2010). Potentially, environmental stress "gets under the skin" not only affecting the HPA-axis (e.g., Kudielka et al., 2009; Loman \& Gunnar, 2010; Quellet-Morin et al., 2011) and/or behavioral functioning (e.g., Anthony et al., 2005; McCarty, Zimmerman, Digiuseppe, \& Christakis, 2005; Pachter, Auinger, Palmer, \& Weitzman, 2006) but also the associations between both

A specific factor affecting stress in children's social environment may be stress in the family environment in the form of maternal parenting stress. Maternal parenting stress may affect the child's daily environment by shaping maternal behavior. Indeed, parenting stress is associated with lower self-reported parenting quality, as evidenced by, for example, more parental laxness, stricter discipline, less nurturing behavior, and lower expectations of the child (Anthony et al., 2005; Guajardo, Snyder, \& Petersen, 2009). Moreover, in these studies parenting stress was also associated with more internalizing and externalizing behaviors, and less social competence in children.

The goal of the current study was to extend the above described knowledge by exploring associations between cortisol stress responses and behavioral functioning at school in a non-clinical sample of 6-year-old children. Additionally, the moderating role of stress in the family environment, operationalized as maternal feelings of parenting stress was investigated. The age of six was chosen because at this age, due to formalization of evaluation at school in many countries, as well as the use of impression management and the development of relief and regret, children may become more exposed and vulnerable to social evaluative stress (e.g., Dickerson \& Kemeny, 2004; Engelmann, Herrmann, \& Tomasello, 2012; Weisberg \& Beck, 2012). To determine the cortisol stress response an innovative, effective (i.e., triggering a significant increase in cortisol), age-appropriate social evaluative stressor was used (de Weerth, Zijlmans, Mack, \& Beijers, 2013). Finally, two indices of cortisol stress responses were calculated: total stress cortisol (i.e., including baseline/anticipation, stressor, and recovery periods), and cortisol stress reactivity (i.e., change in response to the stressor).

\section{2 | METHODS}

\section{1 | Participants}

This study was part of an ongoing longitudinal project that started during pregnancy and focuses on the psychobiological factors associated with children's development (BIBO project; Radboud University). The project and the current cross-sectional data collection were approved by the Institutional Ethical Committee, which follows the Helsinki Declaration (ECG 300107 and ECG 22111/130112, respectively). Participants in the total project were 220 healthy born children and their mothers of whom 193 were still in the project 3 months postpartum (for details, see Beijers, Jansen, Riksen-Walraven, \& de Weerth, 2011; Beijers, Riksen-Walraven, \& de Weerth, 2013; Beijers, Riksen-Walraven, Putnam, de Jong, \& de Weerth, 2013). Of this group, the 188 dyads who were still in the study around the child's 6th birthday were invited to participate in the current data collection. Of the invited group, 149 children participated in a school visit after their parents gave informed consent $\left(M_{\text {age }}=6.09 ; S D=.14 ; M i n=5.87, \operatorname{Max}=6.85 ; 70\right.$ girls $)$. Reasons for non-participation were: child or school preferred not to participate $(n=4)$, family had moved abroad $(n=3)$, or parents declined to participate for other reasons (e.g., they considered the procedure too challenging for their child, they considered the study as too intensive, or personal reasons, $n=32$ ). Children who did not participate $(n=39)$ did not differ significantly from the participating children in educational level of the mother during pregnancy, age of the mother at delivery, gender of the child, or child age four temperament (Children's Behavior Questionnaire short form; CBQ short form; Putnam \& Rothbart, 2006), all p's > .050.

\section{2 | Procedure}

\subsection{1 | School visit}

Children were tested in the afternoon of a regular school day in a mobile laboratory (van) parked near their school (or home $n=8$ of 149). After entering the mobile lab children participated in the Children's Reactions to Evaluation Stress Test (CREST), a social evaluative stress test during which the child carries out three forced-failure tasks containing elements of unpredictability and uncontrollability in front of a "judge" who evaluates their performance (for details, see de Weerth et al., 2013). The procedure takes $20 \mathrm{~min}$ (task performance and anticipation of the judge's evaluation) and is stressful for 5- to 6-year-old children as indicated by increases in cortisol concentrations (de Weerth et al., 2013). After the procedure thorough debriefing took place, in which the child was shown that the tasks were rigged and was told that he/she had performed well and therefore deserved a present. The child then was allowed to draw and watch movies during a 25 -min recovery phase, followed by $25 \mathrm{~min}$ of unrelated tasks (not described/used in this manuscript).

\section{$2.3 \mid$ Measures}

\subsection{1 | Cortisol}

During the school visit, six saliva samples (C1 - C6) were collected from each child. In line with the original CREST (de Weerth et al., 2013) baseline cortisol concentrations were obtained directly before the CREST (children were asked not eat, drink, or be physically active $30 \mathrm{~min}$ prior to the school visit), and $15 \mathrm{~min}$ after the start of the CREST, C1 and C2, respectively. To measure cortisol concentrations in response to the CREST, C3 and C4 were obtained 25 and 35 min after the start of the CREST, respectively. To measure recovery cortisol 
concentrations, $\mathrm{C} 5$ and $\mathrm{C} 6$ were obtained 50 and $58 \mathrm{~min}$ after the start of the CREST. In order to avoid interference of the cortisol circadian rhythm all test sessions started in the afternoon (Dickerson \& Kemeny, 2004) between $12: 15$ and $15: 15 \mathrm{~h}$. If a child was ill on the planned testing day the school visit was rescheduled.

Saliva was collected using eye sponges (BD Visispeare, Waltham, MA; de Weerth, Jansen, Vos, Maitimu, \& Lemtjes, 2007) which participants had to put in their mouth for approximately $1 \mathrm{~min}$. Saliva samples, obtained by centrifuging the eye sponges, were stored in a freezer $\left(-25^{\circ} \mathrm{C}\right)$. Subsequently, cortisol analyses were carried out at the Laboratory of Endocrinology of the University Medical Center Utrecht. An in-house competitive radio-immunoassay was used with a polyclonal anticortisol-antibody (K7348) and [1,2- $\left.{ }^{3} \mathrm{H}(\mathrm{N})\right]$-Hydrocortisone (PerkinElmer NET396250UC) tracer. The inter- and intra-assay variations were $<10.0 \%$ and the lower limit of detection was $1.0 \mathrm{nmol} / \mathrm{L}$.

\subsection{2 | Behavior at school}

Teachers rated children's behavior on 119 items of the Teacher Report Form (TRF; Achenbach, 1991). Each item was rated on a 3-point scale ( 0 = completely not applicable, 2 = clearly or often applicable). Scores for internalizing and externalizing behavior were derived from the internalizing and externalizing subscales of the TRF. Cronbach's $\alpha$ was 0.88 and 0.93 , respectively, for the internalizing and externalizing scale. To increase the normal distribution of the residuals of the regression analyses, scores for internalizing and externalizing behavior were log transformed.

Teachers rated children's prosocial behavior using a 10-item subscale of the Preschool Social Behavior Questionnaire (PSBQ; Tremblay, Vitaro, Gagnon, Piché, \& Royer, 1992). Each item was rated on a 4 -point scale $(0=$ certainly not characteristic, $3=$ very characteristic). The subscale measures altruistic behavior with peers (Cronbach's $\alpha=0.93$ ). No transformation of scores was necessary to improve the normal distribution of the residuals of the regression analysis.

\subsection{3 | Parenting stress}

Mothers filled in the Dutch version of the Parenting Stress Index (PSI; Abidin, 1983): the Nijmeegse Ouderlijke Stress Index (NOSI; de Brock, Vermulst, Gerris, \& Abidin, 1992), a measure of parental experiences of stress due to the parenting situation. The NOSI has 123 items rated on a 6-point scale ( 1 = completely disagree, 6 = completely agree) measuring the domains of parental competence, role restriction, attachment, depression, health, social isolation, marital relationship, as well as child adaptability/plasticity, acceptability, demandingness, mood, distractibility/hyperactivity, and positive reinforcement. Higher scores represented higher levels of maternal feelings of parenting stress with regard to the studied child. Cronbach's $\alpha$ for this measure was 0.96 .

\subsection{4 | Confounders}

Child gender and educational level of the mother were recorded as potential confounders. To measure mothers' educational level, they were asked to indicate their highest educational level ranging from 1 (primary) to 8 (university), followed by "other." Answers on this last option ( $n=5)$ were re-coded into the closest matching option. Gender was coded as girl (0) or boy (1).

\section{4 | Data preparation}

Of the 149 children, five were excluded from the analyses: one because of large deviations from the standard saliva sampling moments, one because of refusal to participate in the saliva sampling, and three because they used medication that might have influenced their cortisol concentrations. Cortisol concentrations of children who had a cold on the testing day ( $n=7$ of 144) did not differ from those of the other children (all $p$ 's > .050) and were therefore retained in the analyses.

\subsection{1 | Cortisol variables}

In Figure 1, cortisol concentrations ( $\mathrm{nmol} / \mathrm{L}$ ) and standard errors for each of the six sampling moments can be found. To analyze whether the stressor, the CREST, induced an increase from the baseline cortisol concentration (lowest of samples C1 and C2; e.g., de Veld, Riksen-Walraven, \& de Weerth, 2012, 2014; de Weerth et al., 2013) to the peak response concentration (highest of samples C3 and C4; de Weerth et al., 2013), a paired samples $t$-test was conducted. There was a significant difference between baseline $(M=6.06$, $S D=2.70)$ and peak response $(M=7.12, S D=3.79)$ cortisol concentrations, $t(141)=-4.41, p<.001$, indicating that the paradigm induced a significant increase in children's cortisol concentrations.

Two common indices of cortisol stress responses were calculated: cortisol stress reactivity and total stress cortisol concentrations. There was a significant correlation between the baseline cortisol concentration and the peak response concentration, Spearman's $\rho=0.69, p<.001$. Therefore, children's cortisol stress reactivity scores were calculated as the standardized residuals of a regression of the peak response on the baseline score (de Veld et al., 2012; Schuetze, Lopez, Granger, \& Eiden, 2008). Total stress cortisol concentrations across all six samples were calculated as the area under the curve: $A \cup C=(C 2+C 1) \times 15 / 2+(C 3+C 2) \times 10 /$ $2+(C 4+C 3) \times 10 / 2+(C 5+C 4) \times 15 / 2+(C 6+C 5) \times 8 / 2$.

\subsection{2 | Missing data}

Of the 144 retained children, maternal parenting stress, was obtained for 139 children (3.5\% missing) and behavior at school for 124 children (13.9\% missing). Of the single cortisol samples 21 were missing (2.4\%) and total stress cortisol concentrations and

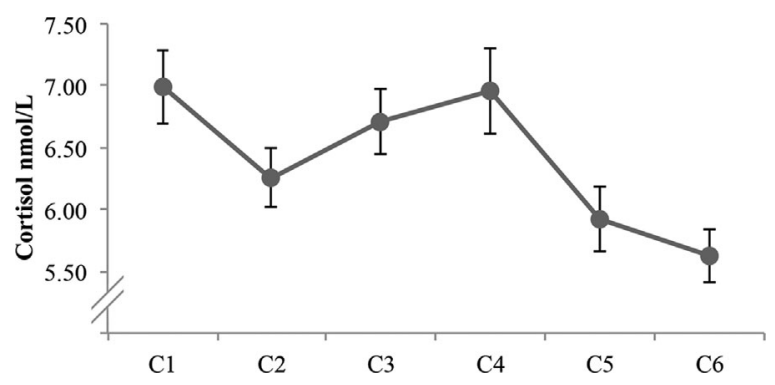

FIGURE 1 Cortisol concentrations (nmol/L) per measurement moment. Error bars represent one standard error above and beneath the mean at each measurement moment 
cortisol stress reactivity were calculated for 134 children $(6.9 \%$ missing) and 142 children (1.4\% missing), respectively.

\section{3 | RESULTS}

\section{1 | Preliminary analyses}

Children tested with the mobile laboratory parked near their home instead of their school (due to the parents' or schools' wishes; $n=7$ of 144) did not differ significantly from the rest on outcome or predictor variables (all $p$ 's > .050). Hence, these children were included in the analyses. Descriptive statistics of the untransformed data are presented in Table 1.

Table 2 presents Spearman correlations of the predictor, outcome, and confounding variables. Higher maternal parenting stress was associated with less prosocial behavior $(\rho=-0.20, p=.030)$. There was a significant correlation between child gender and prosocial behavior $(\rho=-0.25, p=.005)$. Teachers rated girls as more prosocial than boys. Confounders that were significantly associated with an outcome variable were included in the pertaining regression analysis (Tabachnick \& Fidell, 2007). Therefore, gender was included as a confounder in the regression predicting prosocial behavior.

Due to missing outcome or predictor variables 34 of the 144 children dropped out of the main regression analyses. These 34 children did not differ significantly from the other 110 on maternal educational level, gender of the child, or child age four temperament (CBQ short form; Putnam \& Rothbart, 2006), all p's > .050. Mothers of children that dropped out were significantly younger $\left(M_{\text {age }}=37.48\right.$, $S D=4.34)$ than mothers of children remaining in the main analyses $\left(M_{\text {age }}=39.06, S D=3.75\right), t(142)=-2.07, p=.040$.

\section{2 | Main analyses}

A hierarchical regression analysis of prosocial behavior indicated that the first Step (including gender of the child) was significant, $F(1$, $108)=4.05, p=.047$. Gender of the child was significantly associated with prosocial behavior (Table 3 ). Teachers rated boys as less prosocial than girls. Step 2 of this regression (in which the two cortisol stress response indices were added) did not have significant additive value and the model itself was not significant (both $p$ 's > .050). Step 3 (in which maternal parenting stress was added) had significant additive value, $F_{\text {change }}(1,105)=4.31, p=.040$, and the model was significant, $F(4,105)=2.98, p=.023$. Within this model, maternal parenting stress was significantly associated with prosocial behavior (Table 3). Children of mothers with more parenting stress were seen as less prosocial by their teachers. Step 4, (adding the interaction terms between the cortisol stress response indices with maternal parenting stress) did not have significant additive value and the model was not significant (both $p$ 's > .050).

Regarding the other outcome variables (internalizing and externalizing behavior), none of the steps (adding the cortisol stress response indices in Step 1, maternal parenting stress in Step 2, and the interactions between the cortisol stress response indices with maternal parenting stress in Step 3) had significant additive value and none of the models were significant (see Table 3; all p's > .050). In other words, no significant associations were found of the cortisol stress responses, maternal parenting stress, or the interactions between the cortisol stress response indices and maternal parenting stress, with internalizing and externalizing behavior.

\section{4 | DISCUSSION}

This study investigated whether 6-year-old's cortisol stress responses were associated with their behavioral functioning at school. In addition, the moderating role of stress in the family environment, in the form of maternal parenting stress, was investigated. The stress paradigm increased children's cortisol concentrations. However, no support for associations between cortisol stress responses and behavioral functioning or a moderating role of parenting stress was found. Finally, children of mothers with more parenting stress were seen as less prosocial by their teacher.

The finding that the stress paradigm increased children's cortisol concentrations replicated earlier research. De Weerth et al. (2013) showed that the CREST is a valid stressor triggering a modest cortisol stress response in children around the age of 5-6 ( $N=42, M_{\text {age }}=5$ years and 8 months), while leaving room for individual differences. Our replication in a larger and slightly older sample $\left(N=144, M_{\text {age }}=6\right.$ years

TABLE 1 Descriptive statistics of all study variables

\begin{tabular}{|c|c|c|c|c|c|}
\hline & $N$ & $M$ & $S D$ & Min & Max \\
\hline Child gender (\% girls) & 144 & 47.2 & & & \\
\hline Educational level mother & 140 & 6.78 & 1.39 & 3.00 & 8.00 \\
\hline Total stress cortisol (AUC) & 134 & 375.80 & 169.48 & 73.80 & 1474.50 \\
\hline Cortisol stress reactivity $^{a}$ & 142 & 0.00 & 1.00 & -1.77 & 5.15 \\
\hline Parenting stress & 139 & 229.22 & 54.24 & 144.00 & 426.00 \\
\hline Externalizing behavior & 124 & 5.73 & 7.79 & 0.00 & 35.00 \\
\hline Internalizing behavior & 124 & 6.27 & 6.47 & 0.00 & 42.00 \\
\hline Prosocial behavior & 124 & 13.79 & 6.30 & 1.00 & 30.00 \\
\hline
\end{tabular}

AUC, area under the curve (total stress cortisol concentration).

${ }^{\mathrm{a}}$ Due to the operationalization of reactivity as standardized residuals the mean of this variable is 0.00 and the $S D$ is 1.00 . 
TABLE 2 Spearman correlations between all study variables

\begin{tabular}{|c|c|c|c|c|c|c|c|}
\hline & 1. & 2. & 3. & 4. & 5. & $6 .^{a}$ & $7 .^{\mathrm{a}}$ \\
\hline \multicolumn{8}{|l|}{ Confounders } \\
\hline 1. Child gender & - & & & & & & \\
\hline \multicolumn{8}{|l|}{ Predictors } \\
\hline 3. Total stress cortisol (AUC) & -0.04 & -0.05 & - & & & & \\
\hline 5. Parenting stress & 0.05 & 0.14 & -0.02 & -0.07 & - & & \\
\hline \multicolumn{8}{|l|}{ Outcomes } \\
\hline 6. Externalizing behavior ${ }^{a}$ & 0.05 & 0.00 & -0.05 & -0.03 & 0.07 & - & \\
\hline 7. Internalizing behavior ${ }^{a}$ & 0.07 & 0.06 & $-0.18^{+}$ & -0.07 & 0.14 & $0.30^{* *}$ & - \\
\hline 8. Prosocial behavior & $-0.25^{* *}$ & 0.07 & 0.14 & -0.08 & $-0.20^{*}$ & $-0.40^{* * *}$ & $-0.42^{* * *}$ \\
\hline
\end{tabular}

AUC, area under the curve (total cortisol concentration).

a Log transformed.

${ }^{+} p<.100,{ }^{*} p<.050,{ }^{* *} p<.010,{ }^{* * *} p<.001$.

and 1 month) strengthens the case that the CREST is an effective social evaluative stressor for this age group. Moreover, the CREST is considered an ecologically valid stressor for the school environment, given that it consists of social evaluation of the child's performance during challenging tasks, containing elements of unpredictability and uncontrollability. This, together with the paucity of effective stressors for this age group (de Weerth et al., 2013; Gunnar, Talge, \& Herrera, 2009), makes the CREST a promising tool for future stress response research.

The fact that we found no support for associations between cortisol stress responses and behavioral functioning is in line with research that found no associations between cortisol reactivity and mother-reported impulsivity, internalizing, and externalizing behavior of 4.5-year-olds (Spinrad et al., 2009), and with a meta-analysis concluding that cortisol reactivity was not associated with various forms of externalizing behavior in childhood and adolescence (Alink et al., 2008).

Together with these earlier findings, our results may be pointing at a potential dissociation between 6-year-old children's cortisol stress responses and behavioral functioning at school. Although our findings do not warrant this conclusion and more research is needed, speculatively, developmental processes could be behind such a dissociation. The cortisol circadian rhythm appears to continue to develop during childhood and adolescence (Shirtcliff et al., 2012; Simons, Beijers, Cillessen, $\&$ de Weerth, 2015). Similarly, children's cortisol reactions to stressors may still be developing during this period. Hence, children's cortisol stress responses may be less trait-like than those of adults (calibration of stress reactivity; see Boyce \& Ellis, 2005).

Notwithstanding the above, the fact that we found no support for associations between children's cortisol stress responses and behavioral functioning in our study is not in line with earlier findings that cortisol reactivity is associated with self-regulation, student-teacher relationships, school engagement, academic competence, externalizing behavior, aggression, and delinquency (Blair et al., 2005; Conradt et al., 2014; Obradović et al., 2010; Spinrad et al., 2009). Study characteristics may play a role in these apparently contradictory findings.

First, previous studies focused on various aspects of behavioral functioning that associated differently to cortisol responses. For example, Spinrad et al. (2009) reported associations of cortisol reactivity with effortful control, but not with impulsivity, internalizing, and externalizing behavior. This suggests that associations might be behavior-specific.

Second, studies differ in the observers of child behavior. Agreement between teachers and parents regarding child behavior is low to moderate (Winsler \& Wallace, 2002). Behavioral functioning may be situation-specific, and teachers and parents may report more accurately on child behaviors in the context where they observe them most often (school vs. home). This may explain the differences between our findings with teacher reports, and those of Obradović et al. (2010), using a combined measure of parent, teacher, and self-reports.

Third, studies were conducted in different environments. Our sample was middle class whereas earlier studies examined children in less safe environments (e.g., Blair et al., 2005; Conradt et al., 2014; Obradović et al., 2010; Quellet-Morin et al., 2011). While there is ample evidence that (early life) environment affects the HPA-axis, the brain, and behavior (e.g., Kudielka et al., 2009; Loman \& Gunnar, 2010; Lupien, McEwen, Gunnar, \& Heim, 2009; McCarty et al., 2005; Pachter et al., 2006; Simons et al., 2015), the strength and type of associations between HPA-axis and behavioral functioning may be environmentally specific. This could explain why we found no support for associations whereas associations were found by Blair et al. (2005), Conradt et al. (2014), and Obradović et al. (2010).

The fact that our study was conducted with middle class families also suggests that children went to schools with relatively good educational support. This may have reduced the development of behavioral problems (note that in our study only $10.5 \%$ and $12.9 \%$ of the children scored above the clinical cut-off for internalizing or externalizing problems, respectively; Achenbach, 1991; Verhulst, van der Ende, \& Koot, 1997). Protection from the development of 
TABLE 3 Regression models of cortisol stress responses (cortisol stress reactivity and total stress cortisol concentrations - AUC) and maternal parenting stress on behavioral functioning at school

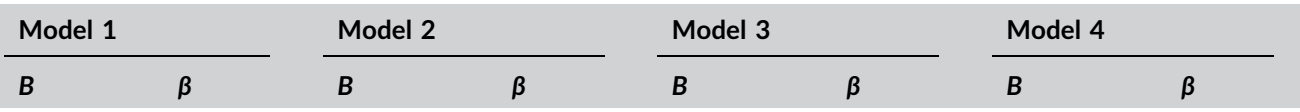

\section{Externalizing behavior}

Step 1

AUC

Reactivity

$<0.01$

0.02

$<0.01$

0.01

$<0.01$

0.06

Step 2

$-0.05$

$-0.12$

$-0.05$

$-0.11$

$-0.07$

$-0.14$

Parenting stress

$<0.01$

0.07

$<0.01$

0.04

Step 3

AUC $\times$ parenting stress

$<-0.01 \quad-0.16$

Reactivity $\times$ parenting stress

$<0.01$

0.01

$R_{\text {change }}^{2}$

0.01

0.01

0.02

$R^{2}$ model

0.01

0.02

0.04

Internalizing behavior

Step 1

\begin{tabular}{|c|c|c|c|c|c|c|}
\hline$A \cup C$ & $<-0.01$ & -0.03 & $<-0.01$ & -0.04 & $<-0.01$ & -0.01 \\
\hline Reactivity & -0.07 & -0.17 & -0.06 & -0.15 & -0.08 & -0.19 \\
\hline \multicolumn{7}{|l|}{ Step 2} \\
\hline Parenting stress & & & $<0.01$ & $0.18^{+}$ & $<0.01$ & $0.17^{+}$ \\
\hline
\end{tabular}

Step 3

AUC $\times$ parenting stress

$<-0.01 \quad-0.06$

Reactivity $\times$ parenting stress

$<0.01$

0.14

$R_{\text {change }}^{2}$

0.03

$0.03^{+}$

0.01

$R^{2}$ model

0.03

$0.07^{+}$

0.08

\section{Prosocial behavior}

Step 1

Child gender

$-2.23$

$-0.19 *$

$-2.39-0.20^{*}$

$-2.08$

$-0.18^{+}$

$-1.97$

$-0.17^{+}$

Step 2

AUC

0.01

$0.19^{+}$

0.01

$0.20^{+}$

0.01

0.17

Reactivity

$-0.96$

$-0.16$

$-1.04$

$-0.17$

$-0.97$

$-0.16$

Step 3

Parenting stress

$-0.02$

$-0.19^{*}$

$-0.02$

$-0.18^{+}$

Step 4

AUC $\times$ parenting stress

$<0.01$

0.08

Reactivity $\times$ parenting stress

$R_{\text {change }}^{2}$

$0.04^{*}$

0.03

$<0.01$

0.01

$R_{\text {model }}^{2}$

$0.04^{*}$

$0.06^{+}$

$0.04 *$

0.01

$0.10^{*}$

$0.11^{+}$

AUC, cortisol area under the curve (total stress cortisol); Reactivity, cortisol stress reactivity. No outliers were removed because Cook's distances indicated no potentially influential data points.

${ }^{+} p<.100,{ }^{*} p<.050$.

behavioral problems may also reduce the formation of a link between behavioral functioning and cortisol stress responses.

The fact that we found no support for moderation by maternal reports of parenting stress is partly in line with Obradović et al. (2010). Although, Obradović et al. (2010) found an interaction effect of the cortisol stress responses and family adversity on prosocial behavior, they did not find this for three other aspects of behavioral functioning; externalizing behavior, school engagement, and academic compe- tence. Although these findings do not indicate the absence of the moderating role per se, they may suggest that family stress does not have a general effect on the association between cortisol stress responses and behavioral functioning. Regarding maternal parenting stress, it might be that it affects the child's experiences of stress in the environment less than expected. In line with this, Anthony et al. (2005) found that the association between parenting stress and child behavior was not mediated by parenting behavior. Stress in the family 
social environment may have more effect when it is more directly aimed at the child. And indeed, Quellet-Morin et al. (2011) found that lower cortisol responses were associated with more behavioral and social (not emotional) problems only in bullied and/or maltreated children.

Alternatively, differences in study characteristics may explain why we found no support for a moderating role of maternal reports of parenting stress. In addition to the characteristics described above, different operationalizations of environmental stress were used. We studied maternal parenting stress. Obradović et al. (2010) and Quellet-Morin et al. (2011) focused on environmental stressors such as financial stress, parenting overload, marital conflict, family expressiveness, maternal depression, maltreatment, and bullying. Some of these factors are also represented in parenting stress in our study (e.g., depression and marital relationship). However, the measures used by Obradović et al. (2010) and Quellet-Morin et al. (2011) also captured more severe forms of stress, potentially affecting the child more, uncovering a link between cortisol stress responses and behavioral functioning.

Interestingly, though not unexpected, children of mothers who experienced more parenting stress were seen as less prosocial by their teacher. This is in line with research indicating that a more adverse environment is associated with children's behavioral functioning (e.g., Conradt et al., 2014; McCarty et al., 2005; Pachter et al., 2006), that more parenting stress is associated with lower levels of child social competence (Anthony et al., 2005), and that parental positivity (positive feelings towards the child and noncoercive parenting) is positively associated with child prosocial behavior (Knafo \& Plomin, 2006).

\section{1 | Strengths, limitations, and future studies}

Study assets were the use of an innovative, age-appropriate, and ecologically valid stressor, which successfully increased cortisol concentrations, as well as the use of biological assessments and two sources of information (i.e., teacher and maternal report). A study limitation was the typical middle class sample as well as the fact that the correlational nature of the data precludes causal conclusions.

Future studies should include broader environmental contexts, also including less safe environments and non-Western cultures to increase generalizability. Moreover, the timing of stress in the environment may be important. Earlier research indicated that stress early in life is associated with HPA-axis functioning of the child (e.g., Kudielka et al., 2009; Loman \& Gunnar, 2010; Quellet-Morin et al., 2011; Simons et al., 2015). Potentially, stress in early life may have a more profound impact than stress later in life, or may have an additive effect upon current stress levels (Essex, Klein, Cho, \& Kalin, 2002).

\section{5 | CONCLUSION}

In this study, children of mothers who experienced more parenting stress displayed less prosocial behavior at school. However, support for associations between 6-year-olds' cortisol stress responses and teacher-reported internalizing, externalizing, and prosocial behavior was lacking, and there was no evidence for moderation by maternal parenting stress.

\section{ACKNOWLEDGMENTS}

We thank the families who kindly participated in the BIBO study. We also thank all research assistants, students, and PhD students, for their assistance with data collection. The present study was originally set up with a personal VIDI grant to Prof. Dr. C. de Weerth (grant 452-04-320) from the Netherlands Organisation for Scientific Research (NWO). It has later been supported with grants of the Behavioural Science Institute, Radboud University, The Netherlands.

\section{CONFLICTS OF INTEREST}

The authors have no conflicts of interest.

\section{REFERENCES}

Abidin, R. R. (1983). Parenting stress index: Manual. Charlottesville, VA: Pediatrics Psycology Press.

Achenbach, T. M. (1991). Manual for the child behavior checklist/4-18. Burlington, VT: Department of Psychiatry, University of Vermont.

Alink, L. R. A., van lizendoorn, M. H., Bakermans-Kranenburg, M. J., Mesman, J., Juffer, F., \& Koot, H. M. (2008). Cortisol and externalizing behavior in children and adolescents: Mixed meta-analytic evidence for the inverse relation of basal cortisol and cortisol reactivity with externalizing behavior. Developmental Psychobiology, 50, 427-450. DOI: 10.1002/dev.20300

Anthony, L. G., Anthony, B. J., Glanville, D. N., Naiman, D. Q., Waanders, C., \& Shaffer, S. (2005). The relationships between parenting stress, parenting behaviour and preschoolers' social competence and behaviour problems in the classroom. Infant and Child Development, 14, 133-154. DOI: $10.1002 /$ icd.385

Beijers, R., Jansen, J., Riksen-Walraven, J. M., \& de Weerth, C. (2011). Attachment and infant night waking: A longitudinal study from birth through the first year of life. Journal of Developmental and Behavioral Pediatrics, 32, 635-643. DOI: 10.1097/DBP.0b013e318228888d

Beijers, R., Riksen-Walraven, J. M., \& de Weerth, C. (2013). Cortisol regulation in 12-month-old human infants: Associations with the infants' early history of breastfeeding and co-sleeping. Stress, 16, 267-277. DOI: 10.3109/10253890.2012.742057

Beijers, R., Riksen-Walraven, J. M., Putnam, S., de Jong, M., \& de Weerth, C. (2013). Early non-parental care and toddler behaviour problems: Links with temperamental negative affectivity and inhibitory control. Early Childhood Research Quarterly, 28, 714-722. DOI: 10.1016/j.ecre sq.2013.06.002

Blair, C., Granger, D., \& Razza, R. P. (2005). Cortisol reactivity is positively related to executive function in preschool children attending Head Start. Child Development, 76, 554-567. DOI: 10.1111/j.1467-8624.2 005.00863.x

Boyce, W. T., \& Ellis, B. J. (2005). Biological sensitivity to context: I. An evolutionary-developmental theory of the origins and functions of stress reactivity. Development and Psychopathology, 17, 271-301. DOI: $10.1017 /$ S0954579405050145

Conradt, E., Abar, B., Lester, B. M., LaGasse, L. L., Shankaran, S., Bada, H., . . Hammond, J. A. (2014). Cortisol reactivity to social stress as a mediator of early adversity on risk and adaptive outcomes. Child Development, 85, 2279-2298. DOI: 10.1111/cdev.12316 
de Brock, A. J. L. L., Vermulst, A. A., Gerris, J. R. M., \& Abidin, R. R. (1992). NOSI/Nijmeegese ouderlijke stress index. Amsterdam: Pearson.

de Veld, D. M., Riksen-Walraven, J. M., \& de Weerth, C. (2012). The relation between emotion regulation strategies and physiological stress responses in middle childhood. Psychoneuroendocrinology, 37, 13091319. DOI: 10.1016/j.psyneuen.2012.01.004

de Veld, D. M., Riksen-Walraven, J. M., \& de Weerth, C. (2014). The relation between gaze aversion and cortisol reactivity in middle childhood. Hormones and Behavior, 65, 173-178. DOI: 10.1016/j.yhbeh.2013.12.012

de Weerth, C., Jansen, J., Vos, M. H., Maitimu, I., \& Lentjes, E. G. (2007). A new device for collecting saliva for cortisol determination. Psychoneuroendocrinology, 32, 1144-1148. DOI: 10.1016/j.psyneuen. 2007.07.005

de Weerth, C., Zijlmans, M. A. C., Mack, S., \& Beijers, R. (2013). Cortisol reactions to a social evaluative paradigm in 5- and 6-year-old children. Stress, 16, 65-72. DOI: 10.3109/10253890.2012.684112

Dickerson, S. S., \& Kemeny, M. E. (2004). Acute stressors and cortisol responses: A theoretical integration and synthesis of laboratory research. Psychological Bulletin, 130, 355-391. DOI: 10.1037/00332909.130.3.355

Engelmann, J. M., Herrmann, E., \& Tomasello, M. (2012). Five-year olds, but not chimpanzees, attempt to manage their reputations. PLoS ONE, 7, 1-7. DOI: $10.1371 /$ journal.pone.0048433

Essex, M. J., Klein, M. H., Cho, E., \& Kalin, N. H. (2002). Maternal stress beginning in infancy may sensitize children to later stress exposure: Effects on cortisol and behavior. Biological Psychiatry, 52, 776-784. DOI: 10.1016/S0006-3223(02)01553-6

Guajardo, N. R., Snyder, G., \& Petersen, R. (2009). Relationships among parenting practices, parental stress, child behaviour, and children's social-cognitive development. Infant and Child Development, 18, 37-60. DOI: $10.1002 /$ icd.578

Gunnar, M. R., Talge, N. M., \& Herrera, A. (2009). Stressor paradigms in developmental studies: What does and does not work to produce mean increases in salivary cortisol. Psychoneuroendocrinology, 34, 953-967. DOI: 10.1016/j.psyneuen.2009.02.010

Knafo, A., \& Plomin, R. (2006). Parental discipline and affection and children's prosocial behavior: Genetic and environmental links. Journal of Personality and Social Psychology, 90, 147-164. DOI: 10.1037/0022-3514.90.1.147

Kudielka, B. M., Hellhammer, D. H., \& Wüst, S. (2009). Why do we respond so differently? Reviewing determinants of human salivary cortisol responses to challenge. Psychoneuroendocrinology, 34, 2-18. DOI: 10.1016/j.psyneuen.2008.10.004

Loman, M. M., \& Gunnar, M. R. (2010). Early experience and the development of stress reactivity and regulation in children. Neuroscience and Biobehavioral Reviews, 34, 867-876. DOI: 10.1016/j.neub iorev.2009.05.007

Lupien, S. J., McEwen, B. S., Gunnar, M. R., \& Heim, C. (2009). Effects of stress throughout the lifespan on the brain, behaviour and cognition. Nature Reviews Neuroscience, 10, 434-445. DOI: 10.1038/nrn2639

McCarty, C. A., Zimmerman, F. J., Digiuseppe, D. L., \& Christakis, D. A. (2005). Parental emotional support and subsequent internalizing and externalizing problems among children. Journal of Developmental and Behavioral Pediatrics, 26, 267-275. DOI: 10.1097/00004703-200508000-00002

Obradović, J., Bush, N. R., Stamperdahl, J., Adler, N. E., \& Boyce, W. T. (2010). Biological sensitivity to context: The interactive effects of stress reactivity and family adversity on socioemotional behavior and school readiness. Child Development, 81, 270-289. DOI: 10.1111/j.14678624.2009.01394x
Pachter, L. M., Auinger, P., Palmer, R., \& Weitzman, M. (2006). Do parenting and the home environment, maternal depression, neighborhood, and chronic poverty affect child behavioral problems differently in different racial-ethnic groups?. Pediatrics, 117, 1329-1338. DOI: 10.1542/ peds.2005-1784

Putnam, S. P., \& Rothbart, M. K. (2006). Development of short and very short forms of the Children's Behavior Questionnaire. Journal of Personality Assessment, 87, 102-112. DOI: 10.1207/s15327752jp a8701_09

Quellet-Morin, I., Odgers, C. L., Danese, A., Bowes, L., Shakoor, S., Papadopoulos, A. S., ... Arseneault, L. (2011). Blunted cortisol responses to stress signal social and behavioral problems among maltreated/bullied 12-year-old children. Biological Psychiatry, 70, 1016-1023. DOI: 10.1016/j.biopsych.2011.06.017

Schuetze, P., Lopez, F., Granger, D. A., \& Eiden, R. D. (2008). The association between prenatal exposure to cigarettes and cortisol reactivity in 7-month-old infants. Developmental Psychobiology, 50, 819-834. DOI: 10.1002/dev.20334

Shirtcliff, E. A., Allison, A. L., Armstrong, J. M., Slattery, M. J., Kalin, N. H., \& Essex, M. J. (2012). Longitudinal stability and developmental properties of salivary cortisol levels and circadian rhythms from childhood to adolescence. Developmental Psychobiology, 54, 493-502. DOI: 10.10 02/dev.20607

Simons, S. S. H., Beijers, R., Cillessen, A. H. N., \& de Weerth, C. (2015). Development of the cortisol circadian rhythm in the light of stress early in life. Psychoneuroendocrinology, 62, 292-300. DOI: 10.1016/j.psyne uen.2015.08.024

Spinrad, T. L., Eisenberg, N., Granger, D. A., Eggum, N. D., Sallquist, J., Haugen, R. G., ... Kupfer, A. (2009). Individual differences in preschoolers' salivary cortisol and alpha-amylase reactivity: Relations to temperament and maladjustment. Hormones and Behavior, 56, 133-139. DOI: 10.1016/j.yhbeh.2009.03.020

Tabachnick, B. G., \& Fidell, L. S. (2007). Using multivariate statistics. (5th ed.). Boston, MA: Allyn \& Bacon.

Tremblay, R. E., Vitaro, F., Gagnon, C., Piché, C., \& Royer, N. (1992). A prosocial scale for the Preschool Behaviour Questionnaire: Concurrent and predictive correlates. International Journal of Behavioral Development, 15, 227-245. DOI: 10.1177/01650254 9201500204

Verhulst, F. C., van der Ende, J., \& Koot, H. M. (1997). Manual for the Teacher's Report Form (Dutch translation). Rotterdam, the Netherlands: Department of Child and Adolescent Psychiatry

Weisberg, D. P., \& Beck, S. R. (2012). The development of children's regret and relief. Cognition and Emotion, 26, 820-835. DOI: 10.1080/0269 9931.2011 .621933

Winsler, A., \& Wallace, G. L. (2002). Behavior problems and social skills in preschool children: Parent-teacher agreement and relations with classroom observations. Early Education and Development, 13, 41-58. DOI: 10.1207/s15566935eed1301_3

How to cite this article: Simons SS, Cillessen $\mathrm{AH}$ de Weerth C. Cortisol stress responses and children's behavioral functioning at school. Dev Psychobiol. 2017;59:217-224. https://doi.org/10.1002/dev.21484 